\title{
LA GEOGRAFÍA EN SEGUNDO DE BACHILLERATO Y EN LAS PRUEBAS DE ACCESO A LAS ENSEÑANZAS UNIVERSITARIAS. SITUACIÓN ACTUAL Y NUEVOS PLANTEAMIENTOS EN ARAGÓN
}

\author{
Paloma Ibarra Benlloch'1, Luis Barreiro Bordonaba², \\ José Luis Calvo Palacios ${ }^{3}$, Pilar Cancer Pomar, \\ Antonio Javier Lacosta Aragüés ${ }^{5}$, Mercedes Lasaosa Sánchez ${ }^{6}$, \\ Luis Mayoral Forniés ${ }^{7}$ y Alfredo Ollero Ojeda ${ }^{8}$
}

1 Armonizadora de Geografía (PAEU) de la Universidad de Zaragoza

Dpto. Geografía y Ordenación del Territorio, Universidad de Zaragoza pibarra@unizar.es

2 Comisión de apoyo a la armonización en Geografía de la Universidad de Zaragoza

IES Francisco Grande Covián

3 Comisión de apoyo a la armonización en Geografía de la Universidad de Zaragoza

Dpto. Geografía y Ordenación del Territorio, Universidad de Zaragoza

4 Comisión de apoyo a la armonización en Geografía de la Universidad de Zaragoza

Centro de Profesores y Recursos Juan de Lanuza

5 Comisión de apoyo a la armonización en Geografía de la Universidad de Zaragoza

Dpto. Geografía y Ordenación del Territorio, Universidad de Zaragoza

6 Comisión de apoyo a la armonización en Geografía de la Universidad de Zaragoza Compañía de María "La Enseñanza"

7 Comisión de apoyo a la armonización en Geografía de la Universidad de Zaragoza IES Pablo Gargallo

8 Comisión de apoyo a la armonización en Geografía de la Universidad de Zaragoza

Dpto. Geografía y Ordenación del Territorio, Universidad de Zaragoza

Resumen: Se presenta un análisis crítico sobre la situación de la Geografía en segundo de Bachillerato en Aragón y su relación con las Pruebas de Acceso a los Estudios Universitarios (PAEU), así como los nuevos planteamientos adoptados y trabajos realizados en la Comisión de apoyo a la armonización. Se ha establecido un nuevo modelo de examen, un documento de orientación de contenidos, herramientas y dife-

Recibido: 16-11-10. Aceptado: 2-2-12. 
rentes medidas de coordinación, comunicación y formación. Los primeros resultados han sido positivos.

Palabras clave: Bachillerato, didáctica, Geografía, PAEU, Universidad, Aragón.

\begin{abstract}
Geography in the entrance test to University of Zaragoza: objectives renewed and methodological approaches. We present a critical analysis of the status of Geography in secondary education of Aragon, and its relation to the entrance to University Studies. A support commission has been created to work on new approaches. It has established a new test model, a guidance document, tools and different measures of coordination, communication and training. The first results have been positive.
\end{abstract}

Keywords: higher secondary-education, didactics, Geography, entrance test, university, Aragon.

\title{
1. Introducción
}

La enseñanza de la Geografía en Bachillerato y las pruebas de acceso a las enseñanzas universitarias (en adelante PAEU) han de buscar siempre su complementariedad. Desde varios ámbitos implicados en este proceso (profesorado de los IES, alumnado y profesorado del Departamento) se constata que no se produce del todo en estos momentos. Esta problemática parece que es común a otras comunidades autónomas y puede que los motivos estén en la insuficiente correlación entre los contenidos del currículo oficial de la asignatura de Geografía, el proceso de aprendizaje de los alumnos -relacionado sin duda con la labor docente del profesorado- y los resultados obtenidos en las PAEU. Con el paso de los años, la valoración que en otro tiempo tuvo la Geografía se ha deteriorado, acaso su imagen global en los institutos y colegios, tanto entre el alumnado de Bachillerato como entre los profesores responsables de impartirla. En consecuencia, se hace necesaria una mayor reflexión crítica tanto en el ámbito universitario como en los centros educativos que sin duda necesitará un mayor esfuerzo de coordinación para mejorar la calidad de los procesos de enseñanza y aprendizaje de la Geografía en el Bachillerato y, así, se impulsarán mejoras en los resultados de las PAEU que pongan en valor la materia y la acción coordinada.

En los últimos años se está asistiendo a un proceso de cambio a raíz del nuevo currículo de Bachillerato (Orden de 1 de julio de 2008 del Departamento de 
Educación, Cultura y Deporte del Gobierno de Aragón) y del nuevo formato de las PAEU (Real Decreto 1892/2008). Este momento ha de aprovecharse para detectar con claridad los problemas existentes y tratar de solucionarlos. Para ello, es imprescindible contar con la opinión, experiencia y coordinación de todas las partes implicadas (Universidad, Departamento de Educación, profesorado y alumnado). En consecuencia, habrá de potenciarse una relación fluida que evite anteriores desajustes y situaciones de insatisfacción que impiden alcanzar los objetivos de calidad de la enseñanza deseados por todos.

Ante esta situación y aprovechando la oportunidad que el momento de cambio de las PAEU brindaba, el Departamento de Geografía de la Universidad de Zaragoza en coordinación con la responsable de la armonización de la asignatura en las PAEU, ha impulsado la comunicación entre las partes implicadas mediante diferentes vías.

Entre ellas destaca la creación de la Comisión de apoyo a la armonización en Geografía (que se explica más adelante) con el objetivo prioritario de mejorar el aprendizaje de la Geografía por parte de los alumnos y que ello se traduzca en la mejora de sus resultados en las PAEU.

Además de recabar la opinión del profesorado de la asignatura de Geografía en Bachillerato, se consideró de interés conocer el punto de vista de los alumnos de la licenciatura de Geografía que habían realizado las pruebas de acceso recientemente. Para ello, se mantuvieron reuniones durante el mes de noviembre de 2009 con todos los alumnos de los cinco cursos de la licenciatura de Geografía de la Universidad de Zaragoza. En la tabla 1 se incluyen sus puntos de vista sobre los problemas de la enseñanza de esta asignatura en $2^{2}$ de Bachillerato y de las PAEU, a través de sus experiencias en los diferentes centros educativos aragoneses. En la tabla 2 se indican propuestas de solución expuestas por los propios alumnos.

Tabla 1. Los problemas de la asignatura de Geografía de $2^{\circ}$ de Bachillerato y de las PAEU según los alumnos de la Licenciatura de Geografía de la Universidad de Zaragoza.

\begin{tabular}{|c|}
\hline $\begin{array}{l}\text { Programa excesivamente amplio, no nos daba tiempo a verlo con tranquilidad y trabajar } \\
\text { bien con los procedimientos, a hacer cosas prácticas, que es lo más interesante. "Mucha } \\
\text { cantidad en muy poco tiempo y luego te olvidas de todo". }\end{array}$ \\
\hline $\begin{array}{l}\text { A veces se hacía pesado y era demasiado memorístico. Sólo se leía y subrayábamos el libro, } \\
\text { no bacía falta discurrir nada, "era soporifero". }\end{array}$ \\
\hline $\begin{array}{l}\text { No hay asignaturas de Geografia en Secundaria, no bay un aprendizaje paulatino aunque, } \\
\text { por otro lado, hay cosas que las hemos visto mil veces desde Primaria y ya te aburren. }\end{array}$ \\
\hline $\begin{array}{l}\text { A nuestros profesores les obsesionaba mucho el examen de Selectividad y a nosotros también. } \\
\text { "Sólo se estudia para el examen de Selectividad, no por aprender de verdad". }\end{array}$ \\
\hline
\end{tabular}


Mi profesor sí era de Geografía pero... no estaba muy contento con los planteamientos de la asignatura y pasaba bastante.

Hay confusión con lo que es la Geografia de verdad, a muchos nos daban el 90\% de Geografía Humana porque es lo más parecido a la Historia y se desprecia a la Geografía Física porque les parece difícil, pero a muchos es lo que más nos gusta.

No nos transmiten lo que es la Geografía ni para qué sirve. "Cuando fui a mi instituto con otros ex-alumnos a contar lo que estaba estudiando, todos se rieron cuando dije que Geografia". "Ni se imaginan lo que aprendemos aqui ni lo que se puede hacer después".

En general no se sabe lo que hace un geógrafo, cuando se sabe te gusta mucho más.

Las Ciencias Sociales no son lo mismo que la Geografía, pero nos meten abi. La Geografia es transversal y eso no encaja en los esquemas ya hechos.

Nunca salíamos de excursión de trabajo de campo, que es con lo que más aprendes. Era todo demasiado teórico, echas de menos cosas más prácticas.

No cuadra la base del Bachillerato con lo que luego hace falta en la Universidad.

Tabla 2. Propuestas de los alumnos para aprender más y mejor, que les enseñen más a gusto y que saquen mejores notas en las PAEU.

Que el examen de Selectividad no condicione tanto lo que tenemos que aprender. Sería mejor al revés: que el examen de Selectividad se centre en los contenidos fundamentales, en lo que nos sirva.

Ajustar más el programa para que dé tiempo a darlo bien con las horas de clase que se tienen. Si todo es importante, ¿por qué no se reparte en otra asignatura más? Además, no se ve nada de Geografía desde $3^{\circ}$ de la ESO.

Aprender menos cosas de "memorieta" y trabajar más con las herramientas, que son mucho más interesantes y amenas y te da la impresión de que son más útiles. "Se debería al menos hablar algo de los SIG, por abi va nuestro futuro".

Que se trabaje más interpretando la realidad y relacionando las cosas, mi profesor@ lo hacía genial pero otros compañeros no tuvieron tanta suerte...

Quitar del programa las cosas que hemos visto mil veces y ya te aburren, así también se descargaría el programa.

Que los profesores de Geografía den la Geografia, los de Historia que den la Historia..., seguro que tanto los alumnos como los profesores están más contentos. No entendemos por qué esto es así, no pasa en otras asignaturas.

Hacer salidas de trabajo de campo para ver en el terreno lo que se explica en clase; se pasa bien y se aprende mucho, es lo mejor. 
Los resultados insatisfactorios obtenidos en las PAEU y los escasos conocimientos anteriores de la materia que exhibe el alumnado (el currículo establece que la Geografía Física se estudie en 1ํㅡㄹ la Geografía Humana y Urbana en $2^{\circ}$ y la Geografía Económica en $3^{\circ}$ de ESO, no impartiéndose contenidos geográficos en $4^{\underline{0}}$ de ESO ni en $1^{\circ}$ Bachillerato) fueron argumentos añadidos por el profesorado como causas y obstáculos destacados de la situación actual, que ayudan a entender el considerable y nada deseable desinterés existente hacia esta asignatura en la ESO y en el Bachillerato.

Ya se ha llevado a cabo un primer análisis crítico de las PAEU de Geografía por parte del profesor Climent López (2001), tras su experiencia como armonizador de Geografía de la Universidad de Zaragoza. En el mismo se ponen de manifiesto los puntos débiles del modelo de examen y del programa específico asociado a él. Este modelo se organizaba en tres apartados: definición de términos geográficos, comentario de gráficos, tablas o mapas y desarrollo de un tema del programa. Con escasas variaciones, esta prueba era muy similar a las vigentes en la mayoría de distritos universitarios del Estado (Muñoz Delgado, 2001; Uña Álvarez, 1998). El autor argumenta con razones fundadas que este tipo de examen no facilita el adecuado aprendizaje de lo esencialmente geográfico que es, como ya señalaba Lacoste (1977), el conocimiento y la interpretación del territorio y de las interrelaciones que lo explican. Como consecuencia de esta situación y adaptándose a la nueva normativa que entraba en vigor, el anterior armonizador esbozó una renovación de las PAEU para el distrito de la Universidad de Zaragoza en junio de 2009, en el que se hacía especial hincapié en el establecimiento de relaciones geográficas. Esta necesidad de renovación y cambio ha venido a inscribirse, de forma más amplia, dentro de un proceso de transformación de mayor calado, como es el de la integración del sistema universitario español en el espacio europeo de enseñanza superior (Ministerio de Educación, Cultura y Deporte, 2003). En este proceso, el paso de planteamientos pedagógicos principalmente teóricos a otros más centrados en la práctica (Moreno Jiménez y Marrón Gaite, 1995) debe basarse en la idea central de la capacidad del alumnado para resolver problemas concretos en el contexto del aprendizaje y aplicación de competencias genéricas (Bradford, 2000; Gardiner y Hughes, 2000; Livingstone y Matthews, 2000; Jenkins, 2000; Escalona et al., 2003).

\section{Nuevos objetivos y planteamientos para las PAEU de Geografía en la Universidad de Zaragoza}

Después de asumir la problemática se intenta caminar hacia su solución lo cual ha llevado al planteamiento de dos objetivos fundamentales para la asignatura de $2^{\circ}$ de Bachillerato y las PAEU asociadas a ella:

- Que el alumnado aprenda bien y con satisfacción la Geografía y ello se vea reflejado en sus calificaciones de las PAEU. 
- Que el profesorado imparta con facilidad y agrado la Geografía transmitiendo al alumnado interés por la asignatura.

Para alcanzar estos objetivos, el Departamento de Geografía y Ordenación del Territorio ha impulsado la creación de la ya citada Comisión de apoyo a la armonización en Geografía . Su primera tarea es reforzar la necesaria comunicación y colaboración entre el profesorado de Bachillerato, que está en el aula con los alumnos, y el armonizador de las PAEU, del que depende el examen de la asignatura en selectividad así como con el profesorado del Departamento universitario que puede también colaborar en el logro de los objetivos planteados (hay un apoyo máximo). La Comisión General de las PAEU apoyó la iniciativa y en octubre del 2009 se creó esta Comisión a la armonización en Geografía con la siguiente composición y perfiles:

- Por parte de la Universidad: armonizadora, representante de la dirección del Dpto.de Geografía y Ordenación del Territorio, representante del área de Geografía Física, representante del área de Geografía Humana, representante del área de Análisis Geográfico Regional.

- Por parte del profesorado de Bachillerato: profesorado de bachillerato con especialidad en Geografía o geógrafo, profesorado de bachillerato con especialidad en Historia o historiador, profesorado de bachillerato de Centros Públicos, profesorado de bachillerato de Centros Concertados y profesorado de Centros de Profesores y Recursos (CPR).

\subsection{Nuevo modelo de examen}

La primera labor acometida por la Comisión fue diseñar un nuevo modelo de examen a partir de los planteamientos renovadores del anterior armonizador, el Dr. Eugenio Climent López. Los rasgos fundamentales de este modelo de examen son los siguientes:

- El examen constará de dos opciones similares A y B. Cada opción se compondrá de dos partes.

- La primera parte se basará en la interpretación de las herramientas básicas que son útiles para la comprensión de los hechos y procesos geográficos y que son el apoyo imprescindible para el proceso de enseñanza-aprendizaje adecuado de la Geografía: mapas, gráficos, tablas estadísticas y textos. Al ser el establecimiento de las relaciones geográficas fundamental para la comprensión y explicación de los hechos y procesos geográficos, se presentarán al alumnado una o dos tipos de herramientas que expresen hechos o procesos geográficos diferentes, pero que tengan alguna relación clara entre ellos y que faciliten al alumnado la interpretación de dichas relaciones. Estas herramientas corres- 
ponderán a uno o dos bloques diferentes del currículo aragonés de la asignatura Geografía.

La segunda opción incluirá algún contenido de uno de los bloques no tratados en la otra opción.

- Los alumnos deberán responder a cuatro o cinco preguntas cortas orientadas a comprobar: el manejo adecuado del vocabulario geográfico, la capacidad de interpretación de los hechos y procesos geográficos presentados en cada mapa o gráfico y el establecimiento de relaciones entre los hechos y procesos geográficos representados.

- La segunda parte del examen consistirá en el desarrollo de un tema, preferentemente del bloque que no haya sido considerado en la primera parte. En la redacción del tema, el alumnado deberá demostrar su madurez y capacidad de compresión de los hechos y procesos geográficos así como su capacidad de síntesis y de expresión clara de las ideas.

\subsection{Comunicación y coordinación}

La comunicación y buena coordinación con todo el profesorado de Bachillerato se considera esencial. Por eso se convocó una reunión con el profesorado encargado de la materia en los IES. En ella se invitó a colaborar para lograr los objetivos, se comentó el nuevo modelo de examen y la necesidad de trabajar en Comisión, de la que se informó sobre sus funciones y quiénes la formaban. Para facilitar esa comunicación y el acceso a la información se creó una nueva sección "PAEUGeografía” en la web del Departamento de Geografía y Ordenación del Territorio de la Universidad de Zaragoza (http://geografia.unizar.es/). Esta sección concentra toda la información relativa a las PAEU de Geografía y en ella se va poniendo a disposición del profesorado y del alumnado todo el trabajo de la Comisión de Geografía y de los diversos colaboradores, organizándose en los siguientes apartados:

1. Información general sobre las PAEU de Geografía del curso vigente.

2. Modelo de examen.

3. Documento de orientación de contenidos para el examen de PAEU.

4. Ejemplos de herramientas geográficas para examen de PAEU.

5. Ejemplos de exámenes.

6. Exámenes PAEU y criterios de corrección.

Aunque hay apartados con documentos más definitivos, como el modelo de exámenes, la mayoría de las secciones se van renovando, mejorando y actualizando, fomentándose en todo momento la participación en el proceso tanto de los profesores de Bachillerato como de la Universidad. 


\subsection{El Documento de Orientación de Contenidos}

Representa el eje central dentro del nuevo planteamiento de trabajo de la geografía que buscamos. Sintetiza y organiza la selección de los contenidos que se consideran fundamentales en la asignatura y en los que se basará el examen de las PAEU. Fue, tras el modelo de examen, la tarea más urgente que debió acometer la Comisión. Es un documento destinado a orientar los contenidos sobre los que los alumnos van a ser evaluados en las PAEU, que no sustituye en modo alguno al programa oficial del que es responsable cada profesor en su centro, sino que lo complementa.

La Comisión de apoyo a la armonización generó un primer borrador que se remitió a todo el profesorado de Bachillerato para su revisión crítica y envío de sugerencias que pudiesen mejorarlo. Tras la revisión del documento se redactó su versión definitiva para el curso 2009-2010. El sistema de trabajo adoptado incluye una evaluación de resultados al final de cada curso, para incorporar las mejoras que se estimen convenientes al Documento de Orientación de Contenidos del próximo curso.

El documento se organiza de acuerdo con los bloques y temas establecidos por la normativa general y el currículo aragonés, concretando en cada uno de los temas tres aspectos esenciales: lo que el alumno ha de saber para comentar los hechos y procesos de cada tema (tabla 3), las herramientas básicas que el alumno ha de saber manejar en cada tema (tabla 4) y el enunciado de los temas concretos que pueden aparecer en el examen (tabla 5). El documento recoge al final de cada bloque un listado con relaciones fundamentales para explicar los hechos y procesos geográficos. No es una enumeración de todas las relaciones posibles, sino una selección relevante y fundamental para la comprensión de la realidad geográfica por parte de los alumnos de $2^{\mathrm{o}}$ de Bachillerato (figura 1).

Tabla 3. Conceptos básicos para las PAEU, agrupados por temas del currículo.

\begin{tabular}{|l|l|}
\hline Tema & \multicolumn{1}{|c|}{ Conceptos } \\
\hline 2.2. & $\begin{array}{l}\text { Unidades morfoestructurales: Zócalo hercínico o Macizo Ibérico, cordilleras alpinas y } \\
\text { depresiones terciarias. Componente litológica del relieve (silícea, calcárea, arcillosa, } \\
\text { volcánica). Formas de relieve estructurales: horizontal, plegado, fallado ofracturado. } \\
\text { Formas de modelado: kárstico, en granito, fluvial, en laderas o vertientes, glaciar y } \\
\text { litoral. }\end{array}$ \\
\hline 2.3. & $\begin{array}{l}\text { Tiempo atmosférico, clima, temperatura media, amplitud térmica, precipitación, } \\
\text { aridez, evapotranspiración, continentalidad, baja presión, anticiclón, frente polar, } \\
\text { gota fría. Tipos de clima de España. Vegetación potencial, vegetación real, dominio } \\
\text { eurosiberiano u oceánico, dominio mediterráneo, vegetación edafófila. Formaciones }\end{array}$ \\
\hline
\end{tabular}




\begin{tabular}{|c|c|}
\hline Tema & Conceptos \\
\hline & $\begin{array}{l}\text { vegetales: bosques (de frondosas caducifolias, de frondosas marcescentes, de } \\
\text { frondosas perennifolias, de coníferas) matorral (arbustivo, subarbustivo), pastos y } \\
\text { formaciones de ribera. Pisos bioclimáticos, cliserie de vegetación. }\end{array}$ \\
\hline 2.4 . & $\begin{array}{l}\text { Cuenca hidrográfica, vertiente hidrográfica, red fluvial, cauce. Caudal, caudal } \\
\text { modular, caudal relativo, crecida, estiaje, régimen pluvio-nival, pluvial). Acuífero o } \\
\text { aguas subterráneas, lago, glaciar, área endorreica. }\end{array}$ \\
\hline 2.5. & $\begin{array}{l}\text { Biodiversidad, impacto ambiental, efecto invernadero, cambio climático, clima } \\
\text { urbano, depuración. Espacios Naturales Protegidos. Desarrollo sostenible, } \\
\text { desertificación, contaminación, sobreexplotación. Energías renovables, consumo y } \\
\text { utilización del agua, depuración de aguas, embalse. }\end{array}$ \\
\hline 3.1. & $\begin{array}{l}\text { Globalización, liberalización comercial, balanza comercial, arancel, importación, } \\
\text { exportación, competencia comercial, Organismos Internacionales, Producto Interior } \\
\text { Bruto. Desarrollo / subdesarrollo (centro/periferia), nivel de vida, calidad de vida, } \\
\text { indice de desarrollo humano (IDH). Aldea global, multinacionales, Unión Europea. }\end{array}$ \\
\hline 3.2. & $\begin{array}{l}\text { Mundo rural, multifuncionalidad rural, Política Agraria Común. Cultivos y } \\
\text { ganadería intensivos/extensivos, secano, barbecho, regadio. Mecanización, } \\
\text { envejecimiento agrario y transformaciones recientes del tamaño de las explotaciones } \\
\text { (redimensionamiento). Industria agroalimentaria, comercialización productos } \\
\text { agrarios, agricultura a tiempo parcial, agricultura ecológica, turismo rural, } \\
\text { residencia secundaria. }\end{array}$ \\
\hline 3.3. & $\begin{array}{l}\text { Industria de base, Industria de bienes de equipo, Industria de bienes de consumo, } \\
\text { reconversión industrial, subcontratación, deslocalización, Fuente de energía, } \\
\text { materia prima, polígono industrial, parque tecnológico, I+D. Población activa sector } \\
\text { industrial, PIB. }\end{array}$ \\
\hline 3.4 . & $\begin{array}{l}\text { Terciarización, servicio público, servicios a la producción, externalización. Comercio } \\
\text { interior, comercio exterior, mayorista, detallista, grandes superficies. Teleco- } \\
\text { municaciones. }\end{array}$ \\
\hline 3.5. & $\begin{array}{l}\text { Turismo, excursionismo, pernoctación, oferta y demanda turística, modelo turístico } \\
\text { tradicional, estacionalidad turística. Turismo alternativo: parque temático, turismo } \\
\text { urbano, turismo cultural, ecoturismo, turismo rural. }\end{array}$ \\
\hline 4.1. & $\begin{array}{l}\text { Tasas brutas de: mortalidad, mortalidad infantil, natalidad, fecundidad, crecimiento } \\
\text { vegetativo. Saldo migratorio, rejuvenecimiento, envejecimiento. Población activa / no } \\
\text { activa, población ocupada y parada, sectores económicos. Crecimiento natural o } \\
\text { vegetativo, crecimiento real, inmigración, emigración, explosión demográfica, } \\
\text { densidad de población. }\end{array}$ \\
\hline 4.2. & $\begin{array}{l}\text { Urbano, situación, emplazamiento. PGOU, clasificación del suelo, rehabilitación, } \\
\text { renovación, revitalización. Morfología urbana: Plano urbano (irregular/ortogonal), } \\
\text { Estructura urbana: Espacio metropolitano, casco antiguo, ensanche, centro de } \\
\text { negocios (CBD), periferia urbana. Ciudad dormitorio, conurbación, megalópolis. }\end{array}$ \\
\hline 4.3. & $\begin{array}{l}\text { Lugar central, área de influencia, macrocéfalo, monocéntrico / policéntrico. } \\
\text { Intermodalidad. Sistema urbano, jerarquía urbana. Red de transporte, trenes de alta } \\
\text { velocidad, redes transeuropeas. }\end{array}$ \\
\hline
\end{tabular}




\begin{tabular}{|l|l|}
\hline Tema & \multicolumn{1}{|c|}{ Conceptos } \\
\hline 4.4. & $\begin{array}{l}\text { Desequilibrio territorial, políticas de ordenación territorial. Fondos estructurales y de } \\
\text { cohesión, FEADER, FSE, LEADER. I+D. }\end{array}$ \\
\hline 4.5. & $\begin{array}{l}\text { Municipio, comarca, provincia, comunidad autónoma, estado. Cortes / parlamento. } \\
\text { Impuestos, Fondo de Compensación Interterritorial. }\end{array}$ \\
\hline
\end{tabular}

Tabla 4. Herramientas básicas para las PAEU, agrupadas por temas del currículo.

\begin{tabular}{|l|l|}
\hline Tema & \multicolumn{1}{c|}{ Lectura e interpretación de: } \\
\hline 2.2. & $\begin{array}{l}\text { 1. Mapa topográfico de la España peninsular } \\
\text { 2. Mapa litológico de la España peninsular } \\
\text { 3. Mapa de unidades morfoestructurales de relieve de la España peninsular }\end{array}$ \\
\hline Clima: 1. Mapas de dominios climáticos en España \\
$\begin{array}{l}\text { 2. Mapa de temperatura media anual de España } \\
\text { 3. Mapa de amplitud térmica de España } \\
\text { 4. Mapa de precipitaciones medias anuales de España } \\
\text { 5. Climogramas de Gaussen (repertorio) É } \\
\text { Vegetación: 1. Mapas de formaciones vegetales en España. } \\
\text { 1.1) Mapa de formaciones vegetales potenciales en España } \\
\text { 1.2) Mapa de formaciones vegetales reales en España } \\
\text { 2. Ejemplos de cliseries de Aragón: Pirineo, Ibérica É }\end{array}$ \\
\hline 1. Mapa de vertientes y cuencas fluviales \\
2. Hidrogramas identificados (repertorio de tipos de regímenes)
\end{tabular}




\begin{tabular}{|c|c|}
\hline Tema & Lectura e interpretación de: \\
\hline 3.4 . & $\begin{array}{l}\text { 1. Gráfico sobre la evolución de población ocupada en sectores productivos } \\
\text { 2. Mapa sobre la participación del sector terciario en la población activa } \\
\text { 3. Textos (repertorio) }\end{array}$ \\
\hline 3.5 . & $\begin{array}{l}\text { 1. Gráfico sobre evolución del número de visitantes y de los ingresos por turismo } \\
\text { 2. Gráfico sobre la estacionalidad del turismo extranjero en España } \\
\text { 3. Mapa o gráfico de turismo internacional por comunidades autónomas } \\
\text { 4. Gráfico de procedencia del turismo extranjero }\end{array}$ \\
\hline 4.1. & $\begin{array}{l}\text { 1. Mapa de densidad de población provincial } \\
\text { 2. Gráfico o tabla sobre la evolución de la natalidad, mortalidad y crecimiento } \\
\text { natural desde los años } \pm 1960-1970 \text { en España } \\
\text { 3. Mapas sobre tasa de natalidad / mortalidad / crecimiento natural actual por } \\
\text { provincias } \\
\text { 4. Gráfico o tabla con la evolución del no de inmigrantes y/o emigrantes en España } \\
\text { en las últimas décadas } \\
\text { 5. Pirámides de edades de España (repertorio desde } \pm 1900 \text { hasta actualidad) } \\
\text { 6. Gráfico de la evolución de la tasa de paro desde las últimas décadas hasta el } \\
\text { momento actual } \\
\text { 7. Gráfico o tabla sobre la evolución por sectores económicos: evolución de la } \\
\text { población ocupada desde el inicio del siglo XX hasta la actualidad }\end{array}$ \\
\hline 4.2. & Plano urbano de Barcelona, Zaragoza, Huesca, Teruel, Valencia, Córdoba. \\
\hline 4.3. & $\begin{array}{l}\text { 1. Mapa del sistema urbano peninsular } \\
\text { 2. Mapa de carreteras de España peninsular } \\
\text { 3. Mapa sobre el tráfico de los principales puertos españoles } \\
\text { 4. Mapa de tráfico de pasajeros y mercancias en los aeropuertos comerciales } \\
\text { 5. Tablas de movimientos de viajeros }\end{array}$ \\
\hline 4.4 . & $\begin{array}{l}\text { 1. Mapa de densidad de población en la Unión Europea } \\
\text { 2. Gráfico y/o tabla sobre evolución del gasto I+D en últimos años de Europa } \\
\text { 3. Mapa del PIB per cápita en la Unión Europea } \\
\text { 4. Texto: (repertorio) }\end{array}$ \\
\hline 4.5 . & 1. Mapa de Comunidades Autónomas y provincias de España \\
\hline
\end{tabular}


Tabla 5. Temas conceptuales a desarrollar por los alumnos en las PAEU.

\begin{tabular}{|c|c|}
\hline Tema & Temas a desarrollar \\
\hline 2.2. & $\begin{array}{l}\text { Tema 1. Los rasgos fundamentales del relieve peninsular: Las grandes unidades } \\
\text { morfoestructurales (zócalo hercinico, cordilleras alpinas y depresiones terciarias) y } \\
\text { los tipos de formas del relieve dominantes. }\end{array}$ \\
\hline 2.3 & $\begin{array}{l}\text { Tema 2. Los grandes dominios bioclimáticos de la España peninsular: Dominio } \\
\text { oceánico y dominio mediterráneo (rasgos climáticos y formaciones vegetales } \\
\text { asociadas). }\end{array}$ \\
\hline 2.5 & $\begin{array}{l}\text { Tema 3. Recursos y demandas hídricas en España (usos, balance hídrico, obras } \\
\text { hidráulicas y política hidráulica). } \\
\text { Tema 4. Los principales problemas medioambientales en España: contaminación } \\
\text { atmosférica y cambio climático; sobreexplotación y contaminación de las aguas; } \\
\text { degradación de la vegetación, erosión y desertificación del suelo; alteración del relieve } \\
\text { (por urbanización y otros usos); residuos urbanos e industriales. Posibles medidas } \\
\text { paliativas de los mismos. }\end{array}$ \\
\hline 3.1 & $\begin{array}{l}\text { Tema 5. Globalización y diversidad: los procesos de mundialización, consecuencias. } \\
\text { Grandes áreas geoeconómicas mundiales y desigualdades. }\end{array}$ \\
\hline 3.2 . & $\begin{array}{l}\text { Tema 6. Dinámicas recientes del mundo rural: transformaciones del mundo rural, } \\
\text { problemática de la actividad agraria en un mundo globalizado, políticas de la Unión } \\
\text { Europea, nuevas orientaciones }\end{array}$ \\
\hline 3.3. & $\begin{array}{l}\text { Tema 7. La actividad industrial en un mundo globalizado; la industria española: } \\
\text { factores de localización y su distribución actual. }\end{array}$ \\
\hline 3.5 & $\begin{array}{l}\text { Tema 8. Principales repercusiones del turismo en España: demográficas, económicas, } \\
\text { territoriales, medioambientales... }\end{array}$ \\
\hline 4.1. & $\begin{array}{l}\text { Tema 9. La población española actual: estructura, evolución y problemática. } \\
\text { Movimientos migratorios desde mediados del siglo XX. }\end{array}$ \\
\hline 4.2 & $\begin{array}{l}\text { Tema 10. La problemática de la vida en las ciudades: problemas derivados de la } \\
\text { planificación urbana, socioeconómicos y ambientales. }\end{array}$ \\
\hline 4.3. & $\begin{array}{l}\text { Tema 11. Los sistemas de transporte terrestres (rasgos generales y problemática) y su } \\
\text { repercusión en la vertebración del territorio a diferentes escalas (Aragón, Península } \\
\text { Ibérica y conexiones con el resto de Europa). }\end{array}$ \\
\hline
\end{tabular}




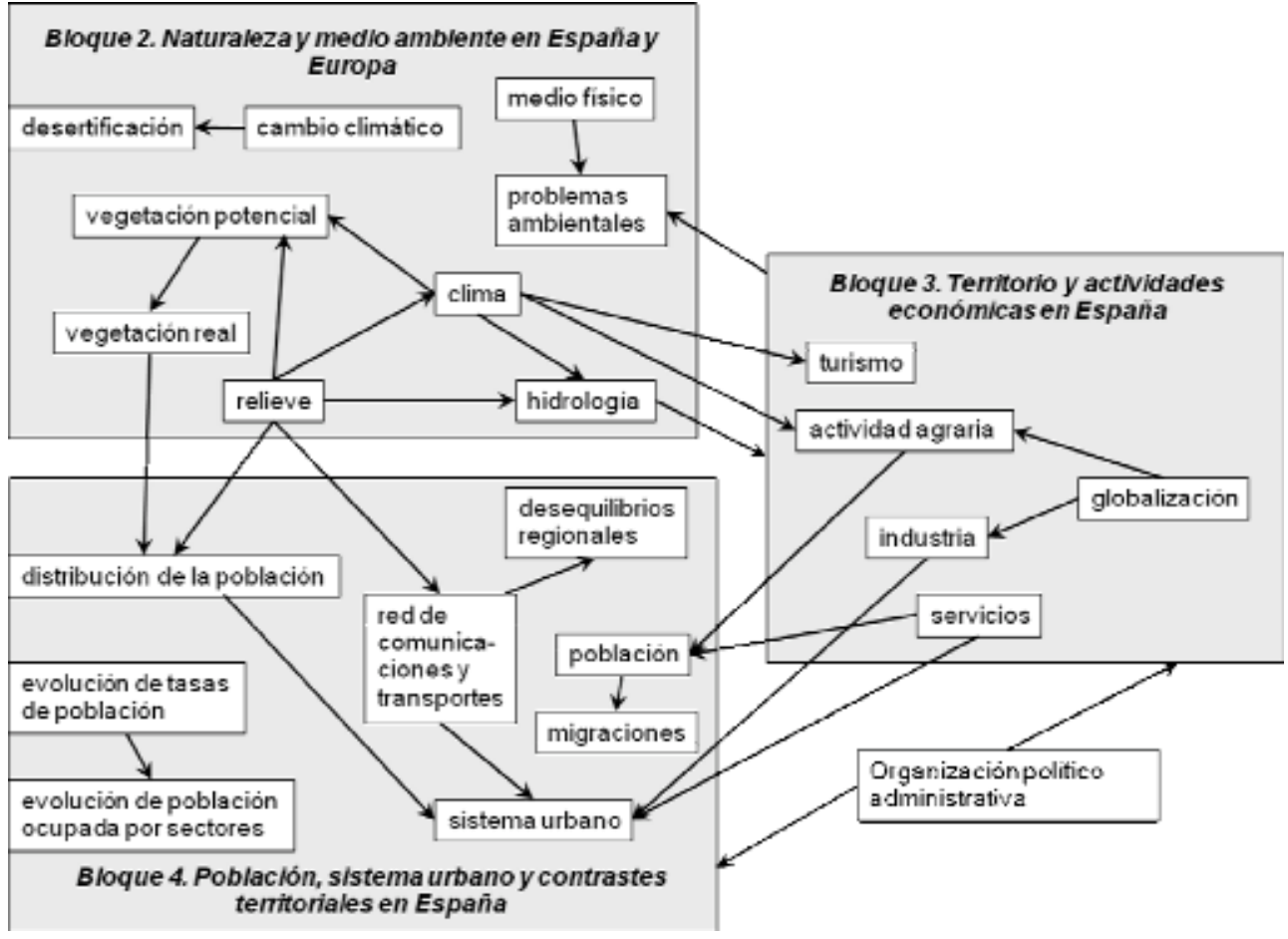

Figura 1. Relaciones geográficas fundamentales para las PAEU.

Como ejemplo se explican a continuación algunas de estas relaciones:

- Clima y vegetación. El clima, a través de temperaturas y precipitaciones, influye decisivamente en la distribución de las formaciones vegetales potenciales. Así, en el dominio bioclimático eurosiberiano se localizan bosques de coníferas (abetales y pinares albares, por ejemplo) y bosques de frondosas caducifolias (hayedos y robledales) adaptados a condiciones más frías y húmedas. En contraste, el dominio bioclimático mediterráneo con menos disponibilidad hídrica posibilita la existencia de bosques de frondosas perennifolias (encinares), bosques de coníferas más adaptadas al estrés hídrico como el pino carrasco o el matorral mediterráneo.

- Relieve y redes de transporte. Es un buen ejemplo de las relaciones en el territorio entre aspectos naturales y humanos, ya que la materia de Geografía de $2^{\circ}$ de Bachillerato permite al alumnado manejar conceptos de bloques temáticos bien diferenciados (el 2, Naturaleza y medio ambiente en España, y el 3, 
Población, sistema urbano y contrastes territoriales en España), y relacionarlos a través de diversas herramientas cartográficas: mapas topográfico, litológico y de unidades morfoestructurales, en el caso del relieve, y mapas de carreteras y tráfico en los principales puertos y aeropuertos españoles, en el caso de las redes de transporte. Analizando esta relación el alumnado puede comprender la complejidad topográfica del territorio, las limitaciones impuestas por el medio a las actividades humanas o la capacidad técnica desarrollada por los seres humanos para superar estos condicionantes, al tiempo que le sirve de soporte para comprender mejor otras realidades territoriales estrechamente vinculadas, como son la distribución de la población, la organización y jerarquía del sistema urbano o los desequilibrios territoriales.

- Servicios y población. La conexión entre población y sector servicios (servicios públicos y a empresas) a partir de los mapas y gráficos seleccionados pretende que el alumnado analice la transformación vivida en España en los últimos 50 años, tanto en el terreno de la demografía como de la economía. Debe tratar los cambios en un terreno y en otro para, conjuntamente, comprender mejor la realidad actual y avanzar cuál puede ser la evolución futura. Para ello se cuenta con mapas y gráficos de población que muestran esa evolución (crecimiento, estancamiento e inmigración) y se relacionan con gráficas de la evolución de los sectores económicos a través de la población ocupada y de la participación del sector terciario en la población activa. El gráfico sobre evolución de la población ocupada en todos los sectores productivos permite ver de forma inmediata el crecimiento del sector servicios, especialmente tras el Plan de Estabilización, pero sin obviar los cambios en los otros sectores.

\subsection{Las herramientas}

La siguiente tarea de la Comisión de apoyo a la armonización fue la selección y/o elaboración de ejemplos de cada una las herramientas establecidas en el Documento de Orientación de Contenidos. Se han intentado presentar en todo momento ejemplos didácticos, fiables y rigurosos, siendo el criterio general el acudir a una fuente de información oficial y actual, siempre que fuera posible y la información fuera del nivel adecuado. En otros casos, se han elaborado los mapas o el material expresamente por profesores del Departamento de Geografía y Ordenación del Territorio, miembros de la Comisión, algunos profesores de Bachillerato y otros colaboradores como profesionales de la Confederación Hidrográfica del Ebro. Esta tarea de revisión crítica y selección ha resultado muy interesante y ha puesto de manifiesto las deficiencias, serias en ocasiones, de algunas herramientas publicadas o difundidas en los materiales didácticos utilizados por los alumnos. 


\subsection{Otras tareas realizadas}

En el curso 2009-2010 se han elaborado 18 ejemplos del nuevo modelo de examen para orientar la preparación adecuada de las PAEU. Los ejemplos fueron preparados por la armonizadora, revisados por los miembros de la Comisión e inmediatamente puestos a disposición de todos en la web citada. La experiencia de poner tantos ejemplos de exámenes diferentes ha sido de gran utilidad para detectar carencias y cuestiones a mejorar.

En cuanto a los exámenes PAEU, hay que destacar el enorme esfuerzo desarrollado para la elaboración de unos criterios de corrección completos y claros que faciliten al máximo la tarea de corrección homogénea en todos los tribunales. Se consideran también fundamentales las reuniones mantenidas entre la armonizadora y todos los correctores con objeto de aunar criterios y lograr la máxima equidad.

Por último, aunque no menos importante de cara al logro de los objetivos planteados, se han propuesto y llevado a cabo una serie de actividades complementarias de apoyo a la docencia de la Geografía en Bachillerato. Estas actividades no tienen una influencia directa en las PAEU pero sí indirecta, pues persiguen facilitar la labor del profesorado de los IES, cubrir algunas carencias que puede tener por diversas razones y, en general, hacerle la asignatura más agradable, didáctica y motivadora para que, en definitiva, el alumnado pueda beneficiarse de ello. Para conseguirlo se llevaron a cabo:

- Realización de itinerarios de campo para $2^{\underline{o}}$ de Bachillerato, impartidos por profesorado del Departamento de Geografía y Ordenación del Territorio en colaboración con los Centros de Formación del Profesorado (CPRs) con el fin de ofrecer al profesorado itinerarios formativos con paradas didácticas, explicaciones y materiales que puedan facilitarle posteriormente la realización de dichas salidas de campo con sus alumnos.

- Selección de recursos didácticos de apoyo útiles para la docencia de la Geografía, puestos a disposición del profesorado en el apartado de materiales docentes de http:/geografia.unizar.es/

- Clases de apoyo al profesorado para la preparación de temas en los que encuentran especial dificultad, como es el caso del relieve.

- Convocatoria del primer concurso anual de logos y lemas de Geografía para alumnos de Bachillerato y Secundaria.

- Firma del acuerdo con el Colegio de Geógrafos de Aragón para la participación de Aragón en la Segunda Olimpiada de Geografía en España.

- Programación de visitas al Departamento y/o a los Centros. Se atienden solicitudes de centros interesados en visitar con sus alumnos el Departamento y 
las instalaciones de la Universidad y solicitudes para que se vaya al centro para hablarles del nuevo grado de Geografía y sus salidas profesionales.

\section{Primeros resultados}

Antes de contar con datos estadísticos de los resultados de las PAEU de Geografía en Aragón en las pruebas de 2010, se recibieron valoraciones en general positivas y de satisfacción por parte del profesorado. El objetivo se ha visto cumplido en los casos en que se ha comunicado que "las calificaciones de mis alumnos a lo largo del curso coinciden con las de las PAEU'. Sin embargo, también hay casos en los que se opina que "los resultados han sido insatisfactorios" por lo que es preciso averiguar las causas y buscar soluciones.

En octubre de 2010 se han recibido los datos de la nota media de la prueba de Geografía, que en junio de 2010 ha sido de 5.88, mientras en junio de 2009 fue de tan sólo 4.50 Habrá que esperar para ver si se confirman estos resultados en los años siguientes para poder concluir si han tenido efectos los cambios metodológicos.

Por otro lado, se ha valorado y agradecido mayoritariamente por parte del profesorado de Bachillerato el trabajo realizado por la Comisión de apoyo a la armonización y la coherencia mostrada. También ha resultado muy positivo el canal de comunicación y colaboración que se ha abierto entre el profesorado de Bachillerato y el profesorado del Departamento de Geografía y Ordenación del Territorio. Ha habido un trabajo colectivo y generoso de muchas personas que sin duda ha supuesto un esfuerzo, pero también ha generado mucha satisfacción.

Parece que se ha logrado comenzar a reducir el escaso interés que provocaba la asignatura de Geografía tanto en los profesores como en los alumnos, por lo que, aunque quedan muchos retos por alcanzar y aspectos a mejorar, se piensa que se está avanzando en la buena dirección. Las opiniones de los alumnos de primer curso del nuevo Grado de Geografía recogidas en septiembre de 2010, transmiten una situación algo más positiva y motivadora que la de los cursos precedentes, siendo también un indicador el muy notable incremento de matrícula registrado en dicho Grado de Geografía en el curso 2010-2011.

\section{Objetivos de futuro}

Los objetivos que siguen planteados son numerosos y requieren el esfuerzo y colaboración de todos:

- Mantener y mejorar la comunicación y coordinación entre las diferentes partes implicadas en el proceso de aprendizaje de la Geografía de los alumnos, con 
especial énfasis en la relación entre el profesorado de Bachillerato, la armonizadora, la Comisión de apoyo, los alumnos y el Departamento de Geografía y Ordenación del Territorio de la Universidad de Zaragoza, pero no en exclusiva. Es importante también la comunicación y coordinación con la Asociación de Geógrafos Españoles, con el colegio profesional, con otros Departamentos de otras comunidades autónomas y con la Administración educativa.

- Analizar con detalle y espíritu crítico los resultados de las PAEU de Geografía en Aragón en cuanto estén disponibles todos los datos estadísticos. Hacer un diagnóstico más certero de la problemática existente.

- Continuar mejorando el Documento de Orientación de Contenidos para las PAEU de manera que se consiga realmente seleccionar aquello fundamental que permita orientar bien el aprendizaje del alumno y la obtención de buenos resultados en las PAEU, acordes a su nivel de madurez y conocimientos.

- Mejorar y ampliar los ejemplos de herramientas para ofrecer al profesor y al alumno herramientas realmente didácticas, actuales y rigurosas, con una adecuada colaboración de los profesionales competentes en la materia.

- Seguir proponiendo nuevos ejemplos de exámenes que apoyen la adecuada preparación de las PAEU con los ejemplos de herramientas seleccionadas.

- Trabajar en la corrección de algunos conceptos o planteamientos equívocos, con el fin de precisar cuestiones erróneas que aparecen con cierta frecuencia en los materiales didácticos utilizados y que dificultan el adecuado proceso de aprendizaje.

- Colaborar en conseguir una adecuada formación del profesorado que debe impartir la asignatura de Geografía en Educación secundaria y Bachillerato, facilitándole y motivándole su labor docente en la medida de las posibilidades.

- Transmitir a los profesores de la asignatura (mayoritariamente no geógrafos) el interés y la utilidad de la Geografía para que puedan a su vez transmitirlo a sus alumnos. Ello, no con el objetivo de provocar vocaciones geográficas, sino de describir su potencialidad y utilidad, sea cual sea la vocación y futuro profesional del alumno.

\section{Referencia}

Bradford, M. (2000). Improving students`'team and personal skills. Gloucester, Geography Discipline Network, Geography \& Environmental Research Unit, and
Cheltenham \& Gloucester College of Higher Education.

Climent López, E. (2001). Las pruebas de acceso a la Universidad y la enseñanza de 
la geografía en el bachillerato español. In: Marrón Gaite, M.J. (ed.) La formación geográfica de los ciudadanos en el cambio de milenio, 611-618, Madrid, Asociación de Geógrafos Españoles, Associaçao de Profesores de Geografía de Portugal y Universidad Complutense.

Escalona, A.; Loscertales, B.; Lardiés, R. y Lacosta, A. (2003). Aportación metodológica para favorecer el aprendizaje de competencias genéricas en la licenciatura de Geografía. In: Marrón Gaite, M.J., Moraleda Nieto, D. y Rodríguez de Gracia, H. (eds.) La enseñanza de la Geografia ante las nuevas demandas sociales, 419-432, Toledo, Asociación de Geógrafos Españoles, Universidad de Castilla-La Mancha y Escuela Universitaria de Magisterio de Toledo.

Gardiner, V. y Hughes, K. (2000). Improving students' problem-solving and thinking skills. Gloucester, Geography Discipline Network, Geography \& Environmental Research Unit, and Cheltenham \& Gloucester College of Higher Education.

Jenkins, A. (2000). The relationship between teaching and research: where does geography stand and deliver? The Journal of Geography in Higher Education, 24(3): 325-351.
Lacoste, Y. (1977). La geografia: un arma para la guerra. Barcelona, Anagrama.

Livingstone, I. y Matthews, H. (2000). Assessing and recording a skills-based curriculum. Gloucester, Geography Discipline Network, Geography \& Environmental Research Unit, and Cheltenham \& Gloucester College of Higher Education.

Ministerio de Educación, Cultura y Deporte (2003). La integración del sistema universitario español en el espacio europeo de enseñanza superior. Documento Marco. Madrid, Ministerio de Educación.

Moreno Jiménez, A. y Marrón Gaite, M.J. (1995). Enseñar Geografía, de la teoría a la práctica, Madrid, Síntesis.

Muñoz Delgado, M.C. (2001). Selectividad. Geografía LOGSE. Pruebas de 2000. Barcelona, Anaya.

Uña Álvarez, E. de (1998). La Geografía en el tránsito de la enseñanza media a la enseñanza superior: experiencia en los grupos de trabajo LOGSE (Galicia). In: Vera Ferre, J.R., Tonda Monllor, E.M. y Marrón Gaite, M.J. (eds.). Educación y Geografía, IV Jornadas de Didáctica de la Geografía, 173-178, Alicante, Asociación de Geógrafos Españoles y Universidad de Alicante. 